\title{
On Some Enigmatic Properties of Relaxation Functions ${ }^{+}$
}

\author{
A. Horzela *, K. Górska and A. Lattanzi \\ Institute of Nuclear Physics IFJ PAN, ul. Radzikowskiego 152, 31-342 Kraków, Poland \\ * Correspondence: Andrzej.Horzela@ifj.edu.pl \\ + Presented at the 37th International Symposium on Dynamical Properties of Solids (DyProSo 2019), Ferrara, \\ Italy, 8-12 September 2019.
}

Published: 5 September 2019

The Debye model of dielectric relaxation provides us with the simplest shape of spectral functions, i.e., relations connecting characteristics of polarized dielectric medium and the frequency of the polarizing electric field. An example of such a relation is $\chi(i \omega)=\left(1+i \omega \tau_{D}^{*}\right)^{-1}$ which shows how the complex susceptibility $\chi(i \omega)$ depends on $\omega$ and on a single material dependent parameter called the characteristic time $\tau^{*}$. As expected, such a spectral function transformed to the time domain leads to the exponential decay law. In contemporary dielectric physics the Debye model finds only a little application and to fit the experimental data it is usually replaced by phenomenologically rooted modifications among which the Cole-Cole, Cole-Davidson, Havriliak-Negami and the "excess wing" models play dominant roles. The first three of them, similarly like the Debye pattern, still depend on a single characteristic time $\tau^{*}$ but involve insertion of fractional powers $0<\alpha, v \leq 1$ according to $\chi(i \omega)=\left(1+\left(i \omega \tau^{*}\right)^{\alpha}\right)^{-v}$. Meanwhile, the "excess wings" model introduces additional characteristic times, e.g. through the ansatz $\chi(i \omega)=\left(1+\left(i \omega \tau_{2}\right)^{\alpha}\right) /\left(1+\left(i \omega \tau_{2}\right)^{\alpha}+i \omega \tau_{1}\right)$. All the above mentioned spectral functions, if treated as functions of a complex variable $\omega \rightarrow z$, share a common behavior - for suitably adjusted, but physically well justified values of parameters, they are analytic in the lower half of the complex plane and map it into the upper half plane. On first sight this property seems negligible but appears to have important mathematical consequences. Functions which obey it have uniquely determined integral representations given as weighted sums of Debye's spectral functions (in mathematics equivalent to the so-called Stieltjes transforms of such weight functions) and, if transformed to the time domain and taken for $t$ $>0$, are completely monotone, i.e., representable as the Laplace transforms of nonnegative locally integrable functions. This means that widely accepted models of non-Debye dielectric relaxation phenomena may be obtained through summing up elementary Debye relaxations with different, continuously distributed characteristic times. In the talk we are going to provide arguments if this statement should be treated either as a mathematical artifact or as a physically based conjecture.

\section{References}

1. Garrappa, R.; Mainardi, F.; Maione, G. Models of dielectric relaxation based on completely monotone functions. Frac. Calc. Appl. Anal. 2016, 19, 1105.

2. Górska, K.; Horzela, A.; Bratek, Ł.; Dattoli, G.; Penson, K.A. The Havriliak-Negami relaxation and its relatives: the response, relaxation and probabilisty functions. J. Phys. A Math. Theor. 2018, 51, 135202. 